\title{
The appearance of the Cornsweet illusion: Measures of perceived contrast and evenness of brightness
}

\author{
RONALD L. GROWNEY \\ University of Connecticut, Storrs, Connecticut \\ and \\ DAVID F. NERI \\ University of Connecticut, Storrs, Connecticut \\ and Naval Submarine Medical Research Laboratory, Groton, Connecticut
}

\begin{abstract}
The appearance of the Cornsweet illusion was assessed quantitatively by obtaining measures of the overall perceived contrast of the illusion stimulus and by obtaining magnitude estimations of the apparent homogeneity/evenness of brightness on either side of the luminance discontinuity, including brightness in the neighborhood of the discontinuity. The perceived broad-area contrast of the illusion matched the physical contrast of a comparison step-function stimulus for physical contrasts of 0.2 and below. Ratings of homogeneity of brightness of the illusion tended to be maximal for those same values of physical contrast.
\end{abstract}

The Cornsweet illusion is one member of a class of perceptual phenomena in which a local perturbation in luminance in an otherwise homogeneous luminance field gives rise to the perception of two uniform fields of different brightnesses, which appear to be divided into the two parts by a sharply defined edge (Cornsweet, 1970; Craik, 1940; O’Brien, 1958; Ratliff \& Sirovich, 1978). The illusion stimulus and its general appearance are depicted in Figure 1. The quality of the appearance of the illusion can be characterized by two major aspects: (1) the extent to which the apparent brightness in the region of the luminance perturbation looks like a step function, sharply defined and homogeneous, with no appearance of the luminance gradients near the discontinuity, and (2) broadarea contrast, or the extent to which the contrast difference across the apparent edge appears to extend over the entire field defined by the illusion stimulus.

Quantitative studies of the illusion have measured only the second aspect of the illusion (Dooley \& Greenfield, 1977; Isono, 1979; Isono, Sakata, \& Kusaka, 1978; Neri,

\footnotetext{
Naval Medical Research and Development Command, Navy Department, Research Work Unit M0100.001-1022. The opinions and assertions contained herein are the private views of the writers and are not to be construed as official or reflecting the views of the Naval Service at large.

We thank Patricia Kramer for comments on this paper. We thank her also for her artful impersonation at the annual meeting of the Optical Society of America, October 1984, where parts of this paper were presented.

The authors' mailing address is: Department of Psychology, University of Connecticut, Box U-20, Storrs, CT 06268.
}

1983). These studies have investigated a wide range of perturbation widths and physical contrasts, only some of which produce an illusion of good quality. In these studies, observers were instructed to vary the contrast of a real edge to match the perceived broad-area contrast of the illusion and to ignore large local contrasts near the apparent edge (i.e., ignore the first aspect). With these instructions, the perceived contrast of the illusion stimulus was found to be equivalent to the physical contrast of a real edge for physical contrasts up to 0.2 (Dooley \& Greenfield, 1977; Isono, 1979; Isono et al., 1978). Furthermore, perceived contrast increases as the width of the perturbation in the illusion stimulus increases for perturbation widths of up to $6^{\circ}$ (Dooley \& Greenfield, 1977) or even $12^{\circ}$ (Isono, 1979) visual angle. At a critical value of physical contrast which depends upon perturbation width, the perceived contrast of the illusion reaches a maximum; with further increases in physical contrast, perceived contrast decreases and the local changes in stimulus luminance become apparent to the observer (Isono et al., 1978).

We addressed three issues in this study. First, we measured quantitatively the first aspect of the illusion (the extent to which the stimulus looks like an ideal step function without local brightness differences in the neighborhood of the luminance discontinuity). Although some observations have been published concerning this aspect of the illusion (Isono et al., 1978), no measurements have been reported. We used magnitude estimation, a rating of the extent to which perceived contrast on either side of the apparent edge appeared even or homogeneous. 


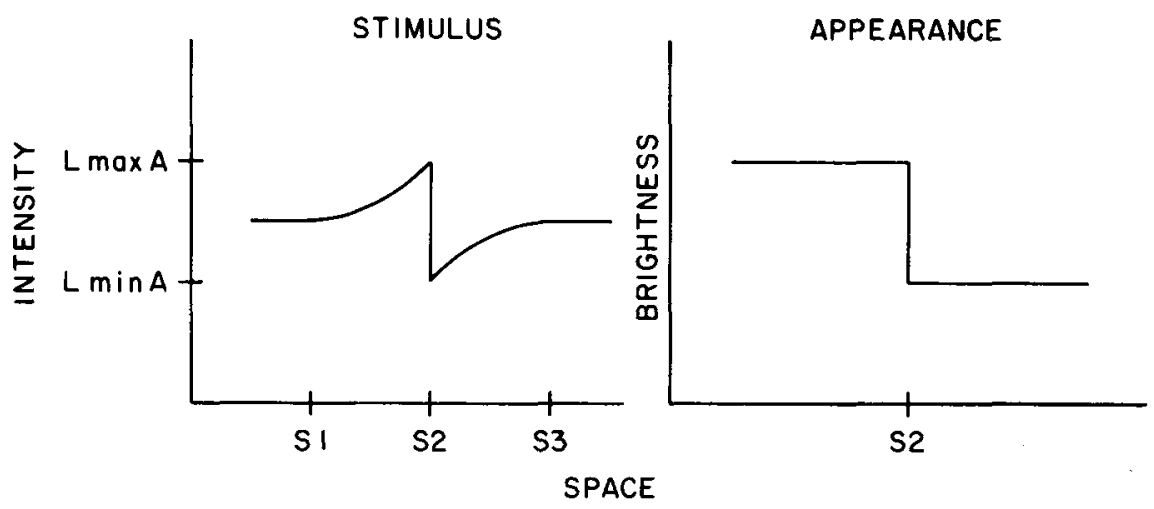

Figure 1. The Cornsweet illusion. The luminance distribution is shown on the left. Perturbation width is defined as the distance $(\mathrm{S3}-\mathrm{S} 1)$ in visual angle. On the right is shown the appearance of the illusion. Some observers have reported Mach-band-like effects near the apparent edge (Arend et al., 1971; Ratliff, 1972). These relatively smaller magnitude effects are not shown here (see Arend et al., 1971).

Second, we measured quantitatively the second aspect of the illusion (broad-area perceived contrast), specifically to address discrepancies in the results of Isono (1979) and Dooley and Greenfield (1977). Isono obtained pronounced decreases in perceived contrast as a function of physical contrast (for values of physical contrast which depended on perturbation width), whereas Dooley and Greenfield obtained quite shallow decreases for similar conditions. One difference in stimuli in the two studies was window width: Isono's was $16^{\circ}$; Dooley and Greenfield's was $4^{\circ}$. A second difference in stimuli was that two of the five perturbation widths used by Dooley and Greenfield had no region of mean luminance at either side; the perturbations either filled the entire window or were wider than the window. These latter two classes of stimulus width, therefore, were not simply illusion stimuli. It is not clear that the illusion was being measured in these two cases because physical contrast differences existed over the entire width of the window. The presence of physical contrast in these two stimuli might have hidden the decrease in perceived contrast which Isono obtained for similar conditions. Therefore, we repeated Dooley and Greenfield's first experiment using a slightly wider window $\left(5^{\circ}\right)$, and we constructed stimuli such that some area of mean luminance bordered each side.

A third purpose of this study was to specify the correspondence between measures of the two aspects; that is, does the measure of the evenness of brightness in the region of the apparent edge vary as a function of the physical contrast in the same way as the measure of broadarea perceived contrast?

\section{METHOD}

\section{Stimuli}

Illusion stimuli were constructed from paper and spun on color wheels. The curved parts of the illusion stimuli were calculated such that the change in luminance over space, as the disks were spun, was parabolic. (See Appendix for details.) The papers used were a white one and a flat black one with a reflectance ratio of 28:1.
The disks, $25.4 \mathrm{~cm}$ in diameter, were $1 / 2$ white and $1 / 2$ black, insuring that the mean luminance of all disks was the same. The contrasts used for these disks were $0.03,0.05,0.1,0.2,0.3,0.4$, or 0.5 . A step-function stimulus that was used for comparison with the illusion stimulus was constructed of the same materials and had a maximum contrast of 0.46 . (Details of contrast determination are given in the Appendix.)

The perturbation widths for these stimuli were $0.2^{\circ}, 0.4^{\circ}, 1.0^{\circ}$, $2.0^{\circ}, 3.5^{\circ}$, or $4.8^{\circ}$ of visual angle. The $0.2^{\circ}$ and $0.4^{\circ}$ perturbation widths were used only with physical contrasts of $0.03,0.05$, and 0.1 , because of difficulty of construction. Therefore, the total number of disks was 34 .

\section{Apparatus}

The step and illusion stimuli were viewed continuously and simultaneously through two channels of a modified three-channel Gerbrands tachistoscope. The configuration was similar to that employed by Dooley and Greenfield (1977), as shown in their Figure 3. Disks containing the step and illusion stimuli were spun at a rate well above flicker fusion. Two rectangular windows, $5^{\circ}$ wide $\times 2^{\circ} 10^{\prime}$ high, were positioned at the ends of the channels and separated vertically by $1^{\circ} 5^{\prime}$, so that the step appeared above the illusion stimulus in the visual field. The third channel of the tachistoscope provided a "balance source" that was required to allow for an alteration in contrast to the step without change in mean luminance. Control of the contrast of the step-function stimulus was accomplished via the use of three polarized filters, one in the path of the step, one in the path of the balance source, and a rotating polarizer in their common path. Luminance variation with polarizer angle did not exceed $10 \%$. The rotating polarizer was turned by a bidirectional dc motor that was controlled by the observer.

The mean luminance in all three channels was $28 \mathrm{fL}$. The illumination was provided by Kodak slide projectors (ELH lamps) and incandescent light bulbs (Sylvania soft white, $40 \mathrm{~W}$ ), positioned to obtain an even distribution of luminance across each window. The maximum variation of luminance within a window measured no more than $4 \%$; the luminance between windows varied $\pm 5 \%$. These luminances were checked weekly using a Spectra Pritchard Model 1970 photometer.

\section{Procedure}

Viewing by the observer was monocular with the left eye. The observer's first task was to match the contrast of the step stimulus to the illusion stimulus by rotating the polarizer. A match was defined as an equal change in brightness from the light to dark halves of both stimuli. As in Dooley and Greenfield (1977), observers were 
instructed to ignore large local differences in brightness in the vicinity of the apparent edge. The method of adjustment was used; the observer made six contrast matches for a particular illusion stimulus by alternating three ascending and three descending trials. These six contrast trials were followed by one magnitude estimation of the evenness of brightness of the illusion stimulus to either side of the apparent edge. For the magnitude estimation task, the observer was instructed to give a number that best represented the homogeneity/evenness of brightness on both sides of the edge. The step-function stimulus, which was continuously in view, was given as an example of homogeneity of brightness and assigned a rating of "10." (A “" 0 " rating was not defined.) After the six contrast matches and one magnitude estimation, a different illusion stimulus was presented. The stimuli were presented in random order. Five complete replications were performed, the first of which was practice. The four experimental replications yielded a total of 24 contrast matches per disk.

\section{Observers}

The observers were two undergraduate students, naive as to the purpose of the experiment. Both observers had normal corrected visual acuity, as measured by a Bausch $\&$ Lomb vision tester.

\section{RESULTS}

\section{Perceived Contrast as a Function of Physical Contrast}

All stimuli produced some amount of broad-area contrast, as shown in Figure 2. The results in this figure replicate the results of Dooley and Greenfield (1977) except for the narrowest stimuli. For stimuli with perturbation widths larger than $1.0^{\circ}$, perceived contrast increased with increasing physical contrast. However, the physical contrast of the illusion stimulus has decreasing effectiveness above a value of 0.2 , as can be seen in the data of both observers. The diagonal line in Figure 2 describes the locus of points for which perceived contrast equals physical contrast; points on this line indicate that the illusion stimulus looks exactly like a real edge of the same physical contrast. With the exception of the two narrowest ones, stimuli with physical contrasts of less than 0.1 had perceived contrasts equal to those of a physical edge with the same contrast. These results are similar to the findings of Dooley and Greenfield (1977) and Isono (1979). For large perturbation-width stimuli with physical contrasts greater than 0.2 , the slope of the perceived contrast function is less than 1.0. The Cornsweet illusion is obtained less strongly as stimulus contrast increases above some minimum.

Above a physical contrast of 0.2 , the perceived contrast of the $1.0^{\circ}$ perturbation-width stimulus not only levels off but decreases noticeably (Figure 2 ). The same result was obtained by Dooley and Greenfield (1977) and Isono (1979). Isono, however, as noted above, obtained a similar decrease for stimuli of all perturbation widths. We hypothesized that Dooley and Greenfield may have failed to obtain such a decrease for all perturbation widths because of their narrower window. However, in this study, we did not observe such decreases for all perturbation widths even though our illusion stimuli did have

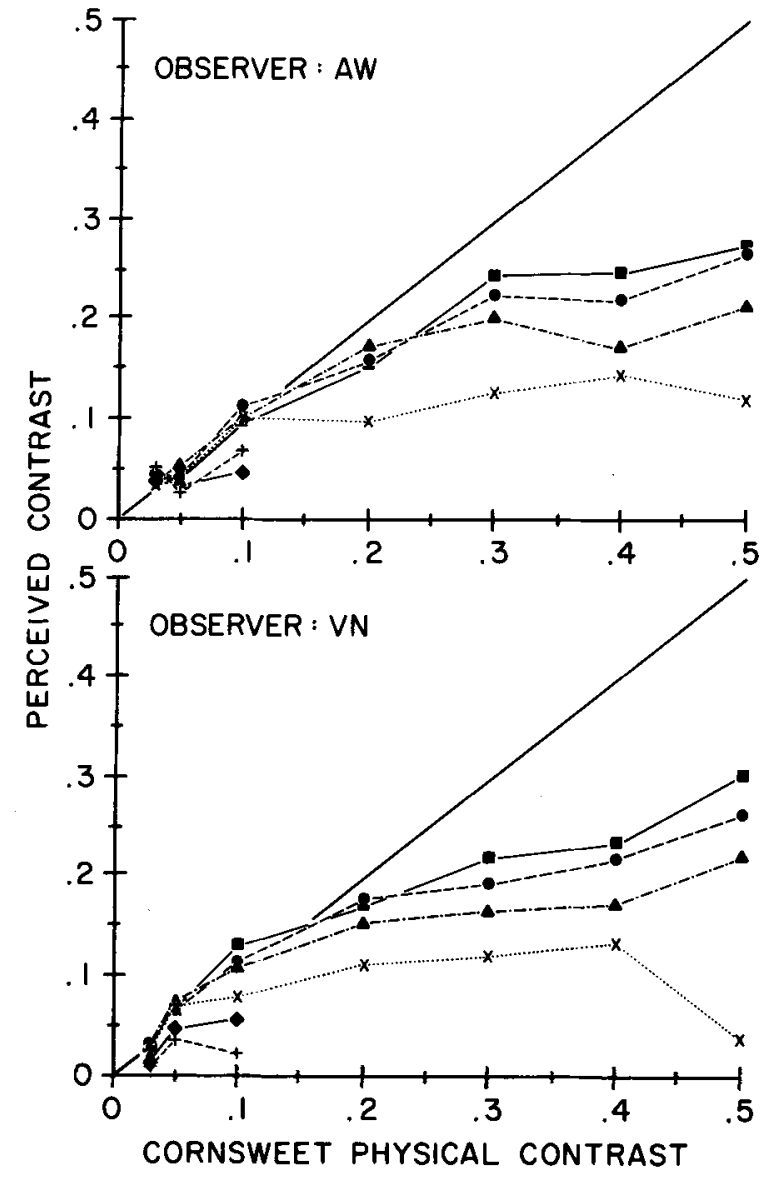

Figure 2. Contrast of the real-edge comparisen stimulus that matched the perceived contrast of the illusion stimulus, as a function of the physical contrast of the illusion stimulus. For perturbation widths of,$+ 0.2^{\circ} ; \diamond, 0.4^{\circ} ; x, 1.0^{\circ} ; \Delta, 2.0^{\circ} ; 0,3.5^{\circ} ; \square, 4.8^{\circ}$. The diagonal line indicates the locus of points for which illusion and real-edge stimuli, which have the same physical contrast, are equal in terms of broad-area perceived contrast. The average standard error for each observer was less than symbol size.

at least small regions of mean luminance at the lateral edges of the window. The similarity of our results to those of Dooley and Greenfield, therefore, suggests that the difference between the results of Dooley and Greenfield and those of Isono is due to some other factor, such as, perhaps, larger regions of mean luminance at the lateral edges of the window or the change in spatial frequency composition with window width (see Discussion).

The narrowest stimuli, $0.2^{\circ}$ and $0.4^{\circ}$ in perturbation width (Figure 2), yielded low, but noticeable, broad-area contrast, which is consistent with informal observations of the stimuli. At a physical contrast of 0.03 , the perceived contrast of both stimuli was equivalent to a real edge of similar physical contrast and was equal in magnitude to the effectiveness of the wider illusion stimuli. This result is consistent with Isono (1979) but not with that of Dooley and Greenfield (1977). ${ }^{1}$ 


\section{Magnitude Estimation as a Function of Physical Contrast}

For physical contrasts above 0.2 , the illusion stimulus appeared decreasingly illusory and increasingly like its luminance distribution. As can be seen in Figure 3, in the data of both observers, the ratings of evenness of brightness on either side of the apparent edge decrease in magnitude as physical contrast increases. This result is consistent with the informal observations of Isono et al. (1978).

The maximum rating of evenness of brightness occurs for at least some value of physical contrast below 0.2 in the data of both observers (see Figure 3). In the data of A.W., all illusion stimuli with physical contrasts less than 0.2 are rated very even in brightness. The data of V.N. show a different pattern for the three widest perturbation widths; stimuli with low values of physical contrast are rated less even in brightness than are stimuli with physical contrasts of 0.1 or 0.2 . A likely explanation of this discrepancy between the data of the two observers, however, may involve the perception by V.N., but not A. W., of Mach-band-like effects; variability of observer report with respect to such effects has been reported before with Cornsweet illusion stimuli (Arend, Buehler, \& Lockhead, 1971; Ratliff, 1972). ${ }^{2}$ Upon debriefing, V.N. reported that he had seen a very narrow line at the apparent edge of the low-contrast stimuli and that he had downrated the evenness of the illusion accordingly; when asked to ignore the narrow line, he rated the stimuli in a manner similar to that of A.W. (those ratings are not shown). A.W., when asked during debriefing, reported that she had not noticed any narrow lines at or near the apparent edge during the experiment.

Although the appearance of the narrow line for some observers may be an important aspect of the illusion, we conclude that the pattern of results shown by $A$.W. best represent the overall appearance of the evenness of brightness of the illusion for values of physical contrast less than 0.2 for the following reasons. First, the apparent Machband-like line is much narrower in spatial extent than even the smallest luminance perturbation width, no more than a few minutes of visual angle. For the greatest part of its spatial extent, then, the illusion appears to be a step function which is homogeneous in brightness. Second, the narrow line is difficult to distinguish from the apparent edge; as noted above, some observers report the effect more than do others, and, in fact, one of the two observers in this study did not report the presence of the narrow line at all. The authors also found the effect to be barely noticeable.

The main conclusion concerning the magnitude estimation data, then, is that the illusion is rated as most similar to a step function in terms of evenness of brightness in the same region of physical contrast where the illusion is perceived as having contrast equal to that of a real edge. Above 0.2 physical contrast, ratings of apparent evenness decrease in the data of both observers.

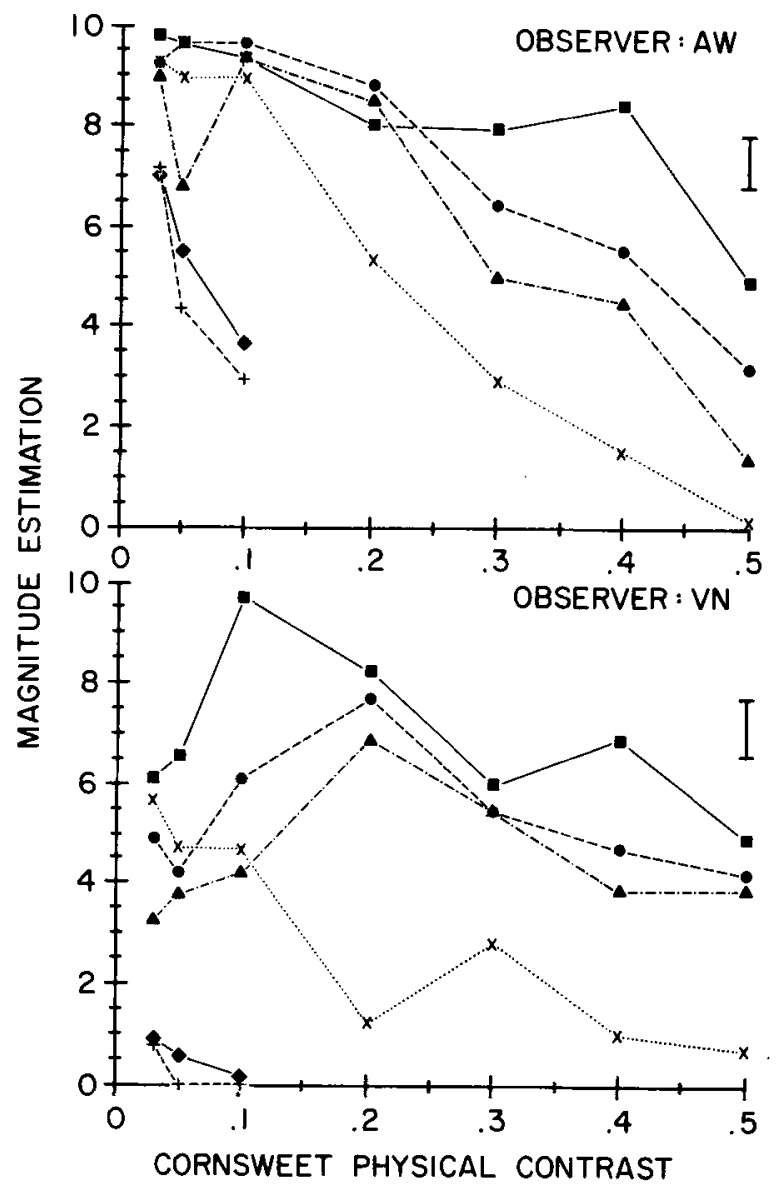

Figure 3. Magnitude estimation ratings as a function of the physical contrast of the illusion stimulus for different perturbation widths. The symbols are as in Figure 2. One average standard error is shown.

\section{DISCUSSION}

Ratings of the homogeneity/evenness of brightness on either side of the apparent edge, including brightness near the edge, covary with perceived (broad-area) contrast. Ratings of evenness were highest for low values of physical contrast $(<0.2)$, the same region of physical contrast for which measures of the illusion's perceived broad-area contrast were equivalent to that of a real edge with the same physical contrast. Furthermore, ratings of evenness decreased as measures of perceived broad-area contrast decreased or became asymptotic. These results are consistent with the notion that measures of perceived broadarea contrast capture the essential aspects of the illusion.

Previous estimates of the physical contrast values that yield the optimum Cornsweet illusion are confirmed by a comparison of the magnitude estimation measures of evenness and the perceived contrast measures. For windows of the size used in this experiment (and also in Dooley \& Greenfield, 1977), the optimal physical contrast to maximize the illusion is between 0.1 and 0.2 for 
perturbation widths in the range of $1^{\circ}$ to $4.8^{\circ}$ of visual angle.

It may be interesting to note that the effect of different window sizes upon the strength of the Cornsweet illusion is consistent, in part, with Dooley and Greenfield's (1977) model, at least at a qualitative level. In the model of Dooley and Greenfield, the effect of increasing physical contrast is to increase the effectiveness of the low spatial frequencies for the system, which effectively emphasizes the difference between the real edge and the illusion stimulus so that the system can distinguish between them. Window size could function analogously to physical contrast in that the larger window has a higher amplitude of low spatial frequency components, which could also emphasize the difference between the real edge and the illusion stimulus. In the data of Isono (1979), perceived contrast dropped off sharply for all the perturbation widths he tested, and did so within regions of physical contrast for which we found no decline. The wider window $\left(16^{\circ}\right)$, then, may have maximized the low spatial frequency content and decreased the strength of the illusion. In our data and those of Dooley and Greenfield, with a narrower window, perceived contrast did not decline, except for the smallest perturbation widths. The smaller window may have helped to maximize the illusion with respect to a wider range of physical contrast. However, this explanation applies only to physical contrasts greater than 0.2 ; below 0.2 , differences due to window width are not observed.

The Dooley and Greenfield (1977) model, as it currently stands, has difficulty, however, in accounting for effects that are not one-dimensional. Todorovic (1983) has constructed a demonstration of the Cornsweet illusion on a CRT in which the local perturbation of luminance, besides being limited horizontally, extends only a few degrees of visual angle vertically; the homogeneous field of luminance, then, completely surrounds the perturbation in two dimensions. In this arrangement, the illusion does not occur; the apparent brightness distribution appears to equal the actual luminance distribution; a vertical fuzzy, bright, and dark perturbation is seen in the middle of the screen. The illusion occurs only when the horizontal edges of the background appear contiguous with the upper and lower extent of the local perturbation. The appearance of the illusion, therefore, seems also to depend upon more global, two-dimensional characteristics of the stimulus display.

\section{REFERENCES}

Arend, L. E., Buehler, J. N., \& Lockhead, G. R. (1971). Difference information in brightness perception. Perception \& Psychophysics, 9, 367-370.

CoRnsweet, T. N. (1970). Visual perception. New York: Academic Press.

CRAIK, K. (1940). Visual adaptation. Unpublished doctoral dissertation, Cambridge University.

Dooley, R. P. , \& GREENFIELD, M. I. (1977). Measurements of edge- induced visual contrast and a spatial-frequency interaction of the Cornsweet illusion. Joumal of the Optical Society of America, 67, 761-765.

Isono, H. (1979). Measurement of edge-induced visual contrast. NHK Laboratories Note, Serial No. 233, 2-11.

Isono, H., SAKaTA, H., \& KUSAKa, H. (1978). Experimental analysis of edge-induced visual contrast phenomenon. Electronics \& Communications in Japan, 61-A, 40-46.

NERI, D. F. (1983). A psychophysical investigation of the Cornsweet effect. Unpublished master's thesis, University of Connecticut, Storrs.

O'BRIEN, V. (1958). Contour perception, illusion and reality. Journal of the Optical Society of America, 48, 112-119.

Raturf, F. (1972). Contour and contrast. Scientific American, 226(6), 91-101.

Ratliff, F., \& Sirovich, L. (1978). Equivalence classes of visual stimuli. Vision Research, 18, 845-851.

Todorovic, D. (1983). Brightness perception and the Craik-O'BrienCornsweet effect. Unpublished master's thesis, University of Connecticut, Storrs.

\section{NOTES}

1. Dooley and Greenfield (1977, their Figure 4) found the $0.1^{\circ}$ and $0.2^{\circ}$ edge width stimuli to be less effective than the corresponding realedge stimulus.

2. Arend et al. (1971), for example, comment that the only variability in the reports of their observers was with respect to Mach-band-like effects at the apparent edge; some of their observers reported the effects more than did others.

\section{APPENDIX}

\section{Illusion Stimuli}

Curve determination. Cornsweet (1970) states that, for the construction of the disks, the average intensity at a point is directly proportional to the relative angular amounts of light and dark that are present at the radial distance of that point from the center of the disk. Let $p$ be the proportion of white at a point, $(x, \theta)$, on the disk, and let $W$ and $B$ be the luminances of the white and black papers, respectively. Then, the average intensity at a point is

$$
L(x, \theta)=W p+B(1-p) .
$$

To obtain a parabolic change in luminance with respect to space, the proportion, $p$, must change parabolically. This is equivalent to $\theta / 360$ changing parabolically; that is,

$$
p=\frac{\theta}{360}=A x^{2}
$$

(see Figure 4; $\theta$ measured in degrees; $x=r 1-K 1$ ). Substituting for $p$ in Equation 1 yields

$$
L(x, \theta)=W\left(A x^{2}\right)+B\left(1-A x^{2}\right)
$$

This relationship describes the curve for the upper spur (the left side of the illusion curve in Figure 4) for values of $x$ between 0 and $(M-K 1)$. The values $(x,-\theta)$ yield the curve for the lower spur (the right side in Figure 4) for values of $x$ between 0 and $(M-K 2)$, where $x=r 2-K 2$. This part of the curve will not look symmetrical to the first part when drawn; $\mathrm{AL}_{2}$ in Figure 4, for example, must be longer than $\mathrm{AL}_{1}$ for the same value of $|x|$ and for corresponding values of $\theta$ and $-\theta$.

Contrast determination. The contrast, $c$, of the illusion stimulus depends on the adjacent maximum and minimum values of $L(x, \theta)$ at the point, $M$. Substituting these values into the equation for Michelson contrast yields 


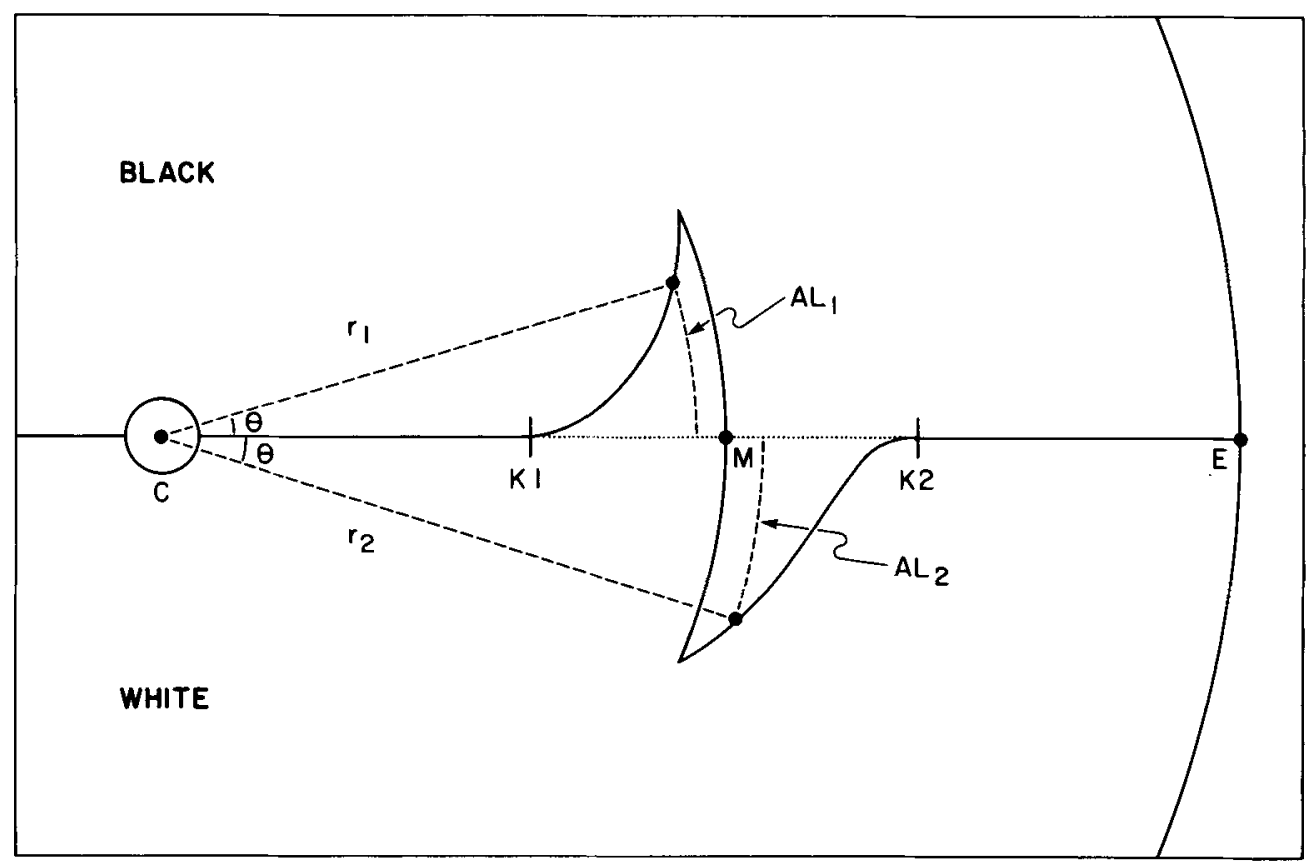

Figure 4. Luminance perturbation for the Cornsweet illusion stimulus. $\mathrm{C}$ denotes the center of the disk, $\mathrm{E}$ the edge. The top half of the disk is black; the bottom half is white. Other details are given in the text.

$$
\mathrm{c}=(2 p-1) \frac{(W-B)}{(W+B)}
$$

\section{Real-Edge Stimulus}

Mean luminance. To make the mean luminance of the realedge stimulus equal to that of the illusion stimulus ( $1 / 2$ white plus $1 / 2$ black) requires an additional step. The construction of the real edge requires putting, for example, more white on the inside portion of the disk to obtain a particular contrast difference. The resulting increase in mean luminance can be balanced by adding a sector of black paper, where the sector width is specified by the angle, $\Phi$ (measured in degrees). For these stimuli, it can be shown that $\Phi$ equals $\theta / 4$.

Starting with a $1 / 2$ white and $1 / 2$ black disk, the real edge can be constructed by adding an additional white portion to the inner part of the disk (radius of $r=M$; Figure 4) of angle, $\theta$, and an additional black sector, equal in radius to the whole disk ( $r=E$; Figure 4), of angle, $\boldsymbol{\Phi}$. Substituting these changed proportions for $p$ in Equation 3 above yields the maximum
(Equation 5) and the minimum (Equation 6) expressions for $L(x, \theta)$ of the real edge:

$$
\begin{aligned}
& L(x, \theta)_{\max }=[W(180+3 \Phi)+B(180-3 \Phi)] / 360, \\
& L(x, \theta)_{\min }=[W(180-\Phi)+B(180+\Phi)] / 360 .
\end{aligned}
$$

These expressions, as well as the following formula for the contrast of the real edge, depend upon the above procedure of adding additional white to the inside portion of the disk. Our equations apply to this case only. The relative luminances, and hence contrast, would change if, for example, black were added to the inside portion.

Substituting Equations 5 and 6 into the equation for Michelson contrast yields the contrast of the real edge,

$$
c=[2 \Phi(W-B)] /[180(W+B)+\Phi(W-B)] .
$$

(Manuscript received August 15, 1985; revision accepted for publication December 18,1985 .) 